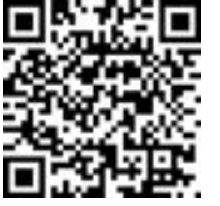

* Médico Cirujano y Partero. Médico Familiar adscrito al Servicio de Atención Médica Continua e Investigador. Asesor del Curso de Especialización en Medicina Familiar del Instituto Mexicano del Seguro Social, avalado por la Universidad de Guadalajara. Maestro en Ética. Doctorante en Bioética y Derechos Humanos, Instituto de Ética y Terapia de Jalisco. ₹ Médico Cirujano y Partero. Médico Residente de la Especialidad en Medicina Familiar.

§ Médico Cirujano y Partero. Especialista en Urgencias Médico Quirúrgicas adscrito al Servicio de Atención Médica Continua.

Unidad de Medicina Familiar número 78 del Instituto Mexicano del Seguro Social. Guadalajara, Jalisco, México.

Correspondencia: ENBP, elinahim@hotmail.es Conflicto de intereses: Los autores declaran que no tienen. Citar como: BecerraPartida EN, Casillas-Torres L, Becerra-Álvarez F. Prevalencia del síndrome coronario agudo en primer nivel de atención. Rev CONAMED 2020; 25(1): 16-22.

doi: 10.35366/92891

Financiamiento: Este trabajo no contó con financiamiento.

Recibido: 04/02/2020. Aceptado: 04/03/2020

\section{Prevalencia del síndrome coronario agudo en primer nivel de atención}

\author{
Prevalence of acute coronary syndrome in primary health care \\ Eli Nahim Becerra-Partida,* Lucía Casillas-Torres, ${ }^{\ddagger}$ Francisco Becerra-Álvarez ${ }^{\S}$
}

\section{RESUMEN}

Objetivo: Determinar la prevalencia de la sospecha y diagnóstico clínico del síndrome coronario agudo (SCA) en la Unidad de Medicina Familiar (UMF) número 78 del Instituto Mexicano del Seguro Social (IMSS), Guadalajara, Jalisco, México. Material y métodos: Estudio realizado en pacientes que acudieron al Servicio de la UMF número 78 del IMSS con sospecha diagnóstica de SCA. Se estratificó por medio de puntajes de TIMI, así como la escala de predicción de riesgo AMR de la Organización Mundial de la Salud. Para la estadística descriptiva se utilizaron frecuencias y proporciones en variables cualitativas; en las cuantitativas media, mediana, moda y desviación estándar. Resultados: De una muestra total de 121 pacientes se descartó SCA en 10.5\%. De los 57 pacientes restantes, se detectó una prevalencia de sospecha y diagnóstico clínico de SCA en la UMF número 78 de 89.5\%; todos ellos presentaron cuadro clínico de dolor torácico y cambios electrocardiográficos. Conclusiones: La prevalencia de sospecha y diagnóstico de SCA en la UMF número 78 en Guadalajara, Jalisco, es de 89.5\%; todos ellos mostraron cuadro clínico de dolor torácico y cambios electrocardiográficos. Estos hallazgos muestran la importancia de priorizar la prevención tratando de bajar el riesgo cardiovascular al disminuir los factores de riesgo modificables y controlar de manera adecuada las patologías existentes, además de mejorar los procesos de identificación clínica, diagnóstico electrocardiográfico, tratamiento en primer nivel de atención y envío oportuno a segundo o tercer nivel.

Palabras clave: Síndrome coronario agudo, primer nivel de atención, isquemia miocárdica.

\begin{abstract}
Objective: To determine the prevalence of suspicion and clinical diagnosis of acute coronary syndrome in the Family Medicine Unit (UMF) number 78 of the Instituto Mexicano del Seguro Social (IMSS) in Guadalajara, Jalisco, Mexico. Material and methods: Study conducted in patients who attended the service of the UMF number 78 of the IMSS with suspected diagnosis of acute coronary syndrome. It was stratified by TIMI scores, as well as the AMR risk prediction scale of the World Health Organization. Frequencies and proportions were used for qualitative variables in descriptive statistics; for quantitative variables average, median, mode and standard deviation. Results: From a total sample of 121 patients, acute coronary syndrome was ruled out of $10.5 \%$. In the remaining 57 patients, a prevalence of suspicion and clinical diagnosis of acute coronary syndrome was found in 89.5\%; all presented a clinical picture of chest pain and electrocardiographic changes. Conclusions: The prevalence of suspicion and diagnosis of acute coronary syndrome in the UMF number 78 in Guadalajara, Jalisco is 89.5\%; all presented a clinical picture of chest pain and electrocardiographic changes. These findings show the importance of prioritizing prevention by trying to lower cardiovascular risk by decreasing modifiable risk factors and adequately controlling existing pathologies. In addition to improving the processes of clinical identification, electrocardiographic diagnosis, treatment in primary health care and timely referral to second or third level of health care.
\end{abstract}

Keywords: Acute coronary syndrome, primary health care, myocardial ischemia. 


\section{INTRODUCCIÓN}

El síndrome coronario agudo se define como un conjunto de entidades clínicas secundarias a la obstrucción brusca del flujo coronario, con el consiguiente desequilibrio entre el aporte de oxígeno y la demanda; esto tiene gravedad y pronósticos variables de acuerdo al área y funcionalidad afectada. Incluye la angina inestable, el infarto agudo al miocardio sin elevación del segmento ST y el infarto agudo al miocardio con elevación del segmento ST?

Según datos de la Organización Mundial de la Salud (OMS), ${ }^{2}$ las enfermedades cardiovasculares son la principal causa de muerte en todo el mundo. Se calcula que en 2012 murieron por esta causa 17.5 millones de personas, lo cual representa 31\% de todas las muertes registradas en el mundo.

La mayoría de las enfermedades cardiovasculares pueden prevenirse actuando sobre los factores de riesgo modificables como el consumo de tabaco, las dietas ricas en carbohidratos y grasas, la obesidad, la inactividad física o el consumo nocivo de alcohol, utilizando estrategias que abarquen a toda la población.

La OMS considera que el cuadro clínico es fundamental para establecer el diagnóstico. Las características de la angina de pecho típicas deben tener una duración mayor de 15 minutos, y tener una intensidad tal que el paciente lo asocie con sensación de muerte. El dolor puede estar irradiado a hombro izquierdo y brazo izquierdo, mandíbula, e incluso región retroesternal y dorso; sin embargo, con el solo hecho de que el dolor se presente en el centro del pecho y tenga dichas características es motivo suficiente para pensar en un síndrome coronario agudo (SCA); una vez diagnosticado, el paciente debe ser observado, evaluado y manejado en una Unidad de Cuidados Coronarios por lo menos durante las siguientes 12 horas. $^{3}$

En el año 2000, la Sociedad Europea de Cardiología y el Colegio Americano de Cardiología redefinieron los criterios de la OMS, los cuales establecían que se requerían dos o más de los siguientes criterios: angina de más de 20 minutos, cambios electrocardiográficos e incremento de los biomarcadores de necrosis. No obstante, el diagnóstico definitivo de SCA se establecía con la presencia de los tres criterios. En esta definición universal del año 2000 se establece que debe haber cierto grado de necrosis (elevación de los biomarcadores, datos clínicos y/o electrocardiográficos). En la tercera revisión de la definición universal en 2012 se establece que debe existir elevación de biomarcadores cardiacos por arriba del percentil 99 del límite normal superior y por lo menos una de las siguientes características: síntomas de isquemia, cambios nuevos o presumiblemente nuevos del segmento ST, bloqueo de rama izquierda del haz de His, desarrollo de Q patológica en el electrocardiograma, evidencia de pérdida del miocardio viable o nuevas alteraciones en la movilidad, evidencia en estudios de imagen de pérdida nueva de miocardio viable o alteraciones nuevas de la contractilidad del miocardio, identificación de trombo intracoronario en la angiografía o autopsia. ${ }^{4-7}$

Existen factores de riesgo asociados al SCA que pueden estar relacionados con la evolución natural de las enfermedades cardiovasculares de acuerdo al estilo de vida o características bioquímicas y fisiológicas; los principales a tomar en cuenta son la hipertensión, diabetes, dislipidemia, tabaquismo, sedentarismo, inactividad física, obesidad, dieta aterogénica, factores psicosociales, así como el consumo de cocaína., 31,-11

El proceso de estratificación de riesgo por medio de la escala de riesgo TIMI es un proceso por el cual se identifican factores de riesgo que modifican el pronóstico de mortalidad temprana. Existe una relación clara entre pronóstico y la puntuación de escala TIMI; sin embargo, aunque logra separar bien los grupos de riesgo, en el individuo concreto tiene una utilidad limitada. ${ }^{12,13}$

En México de acuerdo con las proyecciones del Consejo Nacional de Población de $2010^{14}$ se ha observado que los síndromes isquémicos agudos son la primera causa de muerte en población general. La Organización para la Cooperación y el Desarrollo Económicos (OCDE) en su último reporte de 2013 señaló a México como el país con mayor letalidad por esta patología. ${ }^{15}$

El programa Código Infarto del Instituto Mexicano del Seguro Social es parte de la estrategia integral «A todo corazón», la cual pretende garantizar el diagnóstico y tratamiento al paciente que demanda atención de urgencias por infarto agudo de miocardio, de manera que reciba tratamiento de reperfusión con angioplastia primaria en los primeros 90 minutos o terapia fibrinolítica en los primeros 30 minutos posteriores a su ingreso a los servicios 
de urgencias para de esta manera reducir muertes por dicha patología. Jalisco es el tercer estado que se suma a este proyecto sin precedentes en el sector salud ya establecido en la Ciudad de México y Yucatán, y que emerge como una estrategia prioritaria para contrarrestar el aumento de casos. ${ }^{16}$

Es de gran importancia la sospecha clínica, así como el diagnóstico y tratamiento oportuno en nuestras unidades de medicina familiar que representan el primer contacto del paciente que acude con manifestaciones iniciales de SCA. En la actualidad, no se cuenta con un registro preciso de estos últimos tres años sobre la prevalencia del SCA, por lo que se consideró de suma importancia la realización de este proyecto para conocer la prevalencia de sospecha y diagnóstico en el primer nivel de atención.

\section{MATERIAL Y MÉTODOS}

Se realizó un estudio transversal, descriptivo, prospectivo no analítico con muestreo no probabilístico.

En el universo se consideró a todos los pacientes que acudieron al Servicio de Atención Médica Continua de la UMF número 78 en Guadalajara, Jalisco, México, en un periodo de seis meses comprendido de febrero a julio de 2019, en el que mediante una plantilla de recolección de datos previamente diseñada se solicitó la información requerida como datos de identificación del paciente, datos demográficos, características del dolor torácico, factores de riesgo modificables, factores de riesgo no modificables, todos ellos con pertinencia clínica para el presente estudio. Se realizó la toma de electrocardiograma (EKG) de 12 derivaciones describiendo las características electrocardiográficas, se estableció el tiempo de evolución entre el inicio de los síntomas y el diagnóstico de síndrome coronario agudo, así como las complicaciones que surgieron durante su estancia en dicha unidad médica y si el paciente falleció o no. Además, se estratificó mediante el puntaje de riesgo TIMI y la escala de predicción de riesgo AMR de la Organización Mundial de la Salud y se realizó una revisión de notas médicas con la finalidad de obtener el diagnóstico y los criterios clínicos para el mismo, así como para su seguimiento.

Todos los pacientes con SCA fueron atendidosy se derivaron a su hospital de referencia; para poder dar seguimiento de caso los criterios de inclusión fueron los siguientes: todos los pacientes derechohabientes del IMSS que acudieron al Servicio de Atención Médica Continua con sospecha clínica de SCA, expediente completo y que firmaron consentimiento informado. Se excluyeron los pacientes que no deseaban participar en el estudio, que no contaban con los datos de sospecha clínica de SCA, que no fueran derechohabientes del IMSS y que no firmaran consentimiento informado. El tamaño de la muestra fue de 121 pacientes con sospecha clínica de SCA.

Las variables utilizadas fueron: SCA (que incluye: infarto agudo de miocardio con o sin elevación del segmento ST, angina inestable, elevación de marcadores de necrosis y cuadro clínico), edad, género, antecedente familiar de cardiopatía, antecedente personal de cardiopatía, hipertensión, diabetes, dislipidemia, tabaquismo, obesidad, sedentarismo y consumo de cocaína.

Para el escrutinio de los datos recolectados la información recabada se vació en una hoja del programa Excel de Office versión 2010. Posteriormente se analizaron con el software estadístico SPSS versión 25, utilizando una estadística descriptiva. Una vez que se cumplió el tiempo establecido para este protocolo de estudio se realizó una revisión, verificación y validación final de la base de datos.

En forma global para la estadística descriptiva se utilizaron frecuencias y proporciones en variables cualitativas; en las cuantitativas media, mediana, moda y desviación estándar.

El protocolo fue revisado y aprobado por el Comité Local de Investigación y Ética en Investigación en Salud 1306 de la delegación Jalisco del IMSS, con número de registro R-2019-1306-126. En todo momento se garantizó la confidencialidad de los participantes, el derecho a la no participación y el consentimiento bajo información.

\section{RESULTADOS}

En total se recabaron datos de 121 pacientes, de los cuales cumplieron criterios de inclusión solamente 57, los otros 64 se excluyeron por no contar con los criterios clínicos y electrocardiográficos para el diagnóstico de SCA, los pacientes excluidos presentaron un promedio de edad de 53.5 años, el másjoven fue un paciente de 16 años y el más longevo uno de 89 años; predominó el género femenino con 41 pacientes, 
el masculino con sólo 23 pacientes. Dentro de los factores de riesgo encontramos que 41 pacientes padecían hipertensión arterial, 25 pacientes diabetes mellitus, 23 pacientes contaban con antecedente familiar de síndrome coronario agudo, sólo cuatro reportaron antecedente personal de síndrome coronario agudo, 47 pacientes eran sedentarios, 32 presentaban obesidad, 17 dislipidemia, 18 tabaquismo y sólo uno refirió consumo de cocaína.

Dentro de las causas de dolor torácico en los pacientes que no completaron los criterios de inclusión detectamos diagnósticos de: crisis hipertensiva, costocondritis, hipertensión y diabetes descontroladas, trastorno de ansiedad, taquiarritmias, bradiarritmias, trastorno mixto de ansiedad y depresión, insuficiencia cardiaca, enfermedad pulmonar obstructiva crónica, cáncer de mama, gastritis, enfermedad vascular cerebral y choque anafiláctico. Se hace hincapié en que se observaron más elevados de manera significativa los riesgos cardiovasculares, así como la estratificación de riesgo TIMI en la mayoría de patologías cardiovasculares y metabólicas.

De los 57 pacientes que cumplieron los criterios de inclusión se detectó que 89.5\% efectivamente presentaba un SCA, mismo que se corroboró con la revisión del expediente electrónico después de que el paciente fue atendido en segundo o tercer nivel de atención; sólo en 10.5\% se descartó el síndrome coronario agudo. Cuatro de los pacientes a los cuales se les descartó SCA fueron diagnosticados como crisis hipertensiva, otro con diabetes e hipertensión descontroladasy otro con insuficiencia cardiaca congestiva; sin embargo, todos ellos mostraron cambios electrocardiográficos como supradesnivel o infradesnivel del segmento ST, que junto con la clínica del paciente sugerían un síndrome coronario agudo.

La muestra del estudio estuvo representada en $73.7 \%$ por el género femenino y en $26.3 \%$ por el masculino, de los cuales se encontró una media de edad de 65.02 años, con una mediana de 65 años, una moda de 78 años, con una desviación estándar de 12.033 y una edad mínima de 41 años y máxima de 87 años.

En cuanto al antecedente familiar y personal de SCA se observó que sólo 8.8 y 17.5\% de los pacientes, respectivamente, contaba con dicho antecedente. En la Tabla 7 se muestran los resultados respecto a los factores de riesgo.
Con relación a los cambios electrocardiográficos, se detectó la presencia de elevación en el segmento ST en 23 pacientes (40.4\%), de éstos 23 casos sólo a cinco pacientes (21.73\%) se les realizó trombólisis, misma que fue exitosa en tres pacientes (13.04\%); los otros dos pacientes (8.69\%) tuvieron trombólisis fallida, mismos que fueron sometidos a cateterismo. Otro aspecto importante a tomar en cuenta es que en cuatro pacientes (17.39\%) que mostraron elevación del segmento ST se descartó la presencia de SCA.

Los hallazgos sobre el riesgo cardiovascular calculado con la escala de predicción de riesgo AMR de la Organización Mundial de la Salud se muestran en la Figura 7.

Sobre la estratificación de riesgo TIMI (Tabla 2) se observó que los mayores puntajes estuvieron

\begin{tabular}{lccc}
\multicolumn{4}{c}{ Tabla 1: Factores de riesgo asociados al } \\
SCA, Guadalajara, febrero-julio de 2019. \\
\hline Factor & & $n$ & $\%$ \\
\hline Antecedente & Presente & 5 & 8.8 \\
familiar de SCA & Ausente & 52 & 91.2 \\
Antecedente & Presente & 10 & 17.5 \\
personal de SCA & Ausente & 47 & 82.5 \\
Hipertensión & Presente & 49 & 86.0 \\
arterial sistémica & Ausente & 8 & 14.0 \\
Diabetes mellitus & Presente & 40 & 70.2 \\
& Ausente & 17 & 29.8 \\
Dislipidemia & Presente & 13 & 22.8 \\
& Ausente & 44 & 77.2 \\
Tabaquismo & Presente & 14 & 24.6 \\
& Ausente & 43 & 75.4 \\
Sedentarismo & Presente & 33 & 57.9 \\
& Ausente & 24 & 42.1 \\
Obesidad & Presente & 17 & 29.8 \\
(IMC $\geq$ a 30) 0 - 117 Ausente & 40 & 70.2 \\
Consumo de & Presente & 0 & 0.0 \\
cocaína & Ausente & 57 & 100.0 \\
\hline
\end{tabular}

Abreviaturas: SCA = síndrome coronario agudo; IMC = índice de masa corporal.

Fuente: elaboración propia a partir de los resultados obtenidos en este estudio. 


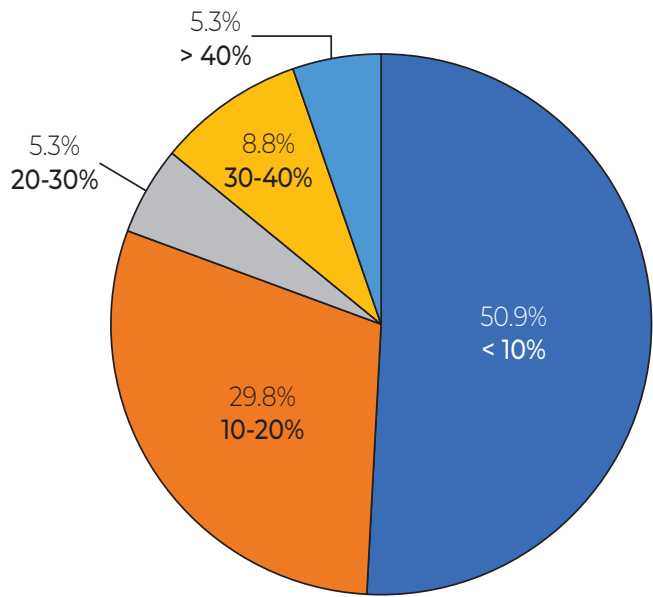

Figura 1: Riesgo cardiovascular de acuerdo con la predicción AMR, Guadalajara, febrero-julio 2019.

Fuente: elaboración propia a partir de los resultados obtenidos en este estudio.

relacionados a síndromes coronarios agudos con elevación del segmento ST, así como a insuficiencia cardiaca congestiva, dicha escala nos predice la probabilidad de muerte a los 30 días.

Con relación al fallecimiento de pacientes, se reportaron dos casos, mismos que ocurrieron fuera de la Unidad de Medicina Familiar número 78, pero se documentaron al realizar la revisión de expedientes, ambos casos corresponden a pacientes del sexo femenino con edades de 78 y 81 años, las cuales contaban con un puntaje TIMI de cuatro y seis puntos.

\section{DISCUSIÓN}

Con base en la tercera definición universal de infarto, todos los pacientes incluidos en el estudio presentaron síntomas sugestivos de isquemia así como cambios electrocardiográficos, también sugestivos de la patología; sin embargo, por ser una clínica de primer nivel no se pudieron realizar las enzimas cardiacas para confirmar o descartar la patología, en su lugar se dio seguimiento mediante el expediente electrónico en el cual pudimos confirmar el diagnóstico sospechado en $89.5 \%$ de los casos y descartarlo en el otro 10.5\%.

Un importante reto continuo de los médicos de urgencias es la sospecha y diagnóstico oportuno y rápido, la herramienta fundamental es la actualización continua, por lo que el programa nacional
Código Infarto del IMSS es pieza clave para la educación continua. Los médicos de urgencias tienen la obligación de sospechar, diagnosticar y tratar al paciente con electrocardiograma de ingreso en menos de 10 minutos, respetar el tiempo puerta aguja para la terapia fibrinolítica del paciente si éste lo amerita en menos de 30 minutos y cumplir con el tiempo puerta balón para reperfusión con angioplastia primaria cardiaca en menos de 90 minutos. El programa Código Infarto del IMSS es un programa exitoso que dará frutos en reducción de mortalidad con mayor prevalencia e incidencia a nivel nacional. ${ }^{16}$

Los hallazgos en esta investigación en cuanto a la mortalidad fue de 3.5\%, una incidencia muy baja a diferencia de lo que menciona la OCDE en el reporte de 2013,15 en la cual indica que México es el país con mayor letalidad por esta patología con una incidencia de $27.2 \%$, muy por encima de la media en el resto del mundo que es de $7.9 \%$; sin embargo, es importante tomar en cuenta que nuestra muestra fue pequeña y que al haberse realizado en una unidad médica de primer nivel es menor la afluencia de pacientes con clínica sugestiva de SCA.

La estrategia Código Infarto pretende garantizar el diagnóstico y tratamiento al paciente que

\section{Tabla 2: Estratificación de riesgo TIMI,} Guadalajara, febrero-julio de 2019.

\begin{tabular}{crr}
\hline Puntaje & $\mathrm{n}$ & $\%$ \\
\hline 0 & 1 & 1.8 \\
1 & 13 & 22.8 \\
2 & 3 & 5.3 \\
3 & 8 & 14.0 \\
4 & 15 & 26.3 \\
5 & 7 & 12.3 \\
6 & 6 & 10.5 \\
7 & 2 & 3.5 \\
8 & 1 & 1.8 \\
9 & 0 & 0.0 \\
10 & 1 & 1.8 \\
Total & 57 & 100.0 \\
\hline
\end{tabular}

Fuente: elaboración propia a partir de los resultados obtenidos en este estudio. 
demanda atención de urgencias por infarto agudo al miocardio, de manera que reciba tratamiento de reperfusión con angioplastia primaria en los primeros 90 minutos o terapia fibrinolítica en los primeros 30 minutos posteriores a su ingreso a urgencias, lo cual disminuye la mortalidad por dicha patología. Constatamos que esta estrategia está funcionando de manera efectiva y exitosa. ${ }^{16}$

Nuestro estudio evidenció la presencia de elevación en el segmento ST en 23 pacientes, de los cuales cinco recibieron tratamiento de trombólisis y en tres fue exitoso; cabe señalar que dichos pacientes acudieron a recibir atención médica en un promedio de dos horas posteriores al inicio de los síntomas. Con base en estos resultados observamos que 39.12\% de los pacientes potencialmente candidatos a trombólisis están recibiendo la atención adecuada en los tiempos que pretende la estrategia Código Infarto. Sería importante constatar si el otro $60.88 \%$ de los pacientes acudió en forma temprana a recibir atención porque posiblemente no se encontraban en ventana trombolítica; sin embargo, en nuestro estudio hubo un subregistro del tiempo de evolución entre el inicio de los síntomas y el diagnóstico, por lo que nos es imposible establecer este dato.

Coincidimos con diversos estudios realizados $^{8,9,17}$ en los cuales se considera una fuerte asociación entre los factores de riesgo y el aumento significativo de riesgo de desarrollar un evento cardiovascular, ya que el presente estudio reveló que $86 \%$ de los pacientes cuenta con antecedente de hipertensión arterial, 70.2\% tiene antecedente de diabetes, $22.8 \%$ con dislipidemia, $24.6 \%$ refiere tabaquismo activo, 57.9\% son sedentarios, 29.8\% padece obesidad y 0\% de los pacientes reportó consumo de cocaína.

Acerca del riesgo cardiovascular calculado con la escala de predicción de riesgo AMR de la Organización Mundial de la Salud nos encontramos con una limitante para el presente estudio, ya que dichas tablas consideran pacientes desde los 40 hasta los 70 años y en nuestra población estudiada detectamos seis pacientes por arriba de 80 años, a los cuales se les consideró dentro del grupo de 70 años para calcular el riesgo cardiovascular, por lo que sugeriríamos modificaciones futuras en estas escalas debido al aumento creciente en la expectativa de vida. Es importante señalar que ésta es una escala diseñada para pacientes que aún no han mostrado síntomas de enfermedad cardiovascular y en quienes aún se pueden tomar medidas de prevención primaria; empero, decidimos aplicarlas en nuestro estudio para valorar la asociación del riesgo cardiovascular, el cual se esperaría elevado en pacientes que ya se encuentran cursando con un evento cardiovascular. Por el contrario, constatamos que entre los pacientes incluidos en el estudio, 50.9\% tienen riesgo menor de 10\%, 29.8\% tienen riesgo de 10 a 20\%, 5.3\% tienen riesgo de 20 a 30\%, $8.8 \%$ tienen riesgo de 30 a $40 \%$ y $5.3 \%$ tienen riesgo mayor de $40 \%$ de padecer un episodio cardiovascular, mortal o no, en un periodo de 10 años, por lo que podríamos inferir que no es un buen factor predictor; no obstante, en los pacientes excluidos del estudio se observó un aumento considerable de riesgo cardiovascular en aquéllos con patologías cardiacas y/o metabólicas.

Encontramos coincidencias con Gines, ${ }^{13}$ ya que nuestro estudio evidenció una asociación fuerte de la estratificación de riesgo TIMI con los síndromes coronarios agudos, así como una elevación mayor del puntaje TIMI en síndromes coronarios con elevación del segmento ST en comparación con el resto de los pacientes; sin embargo, los pacientes finados no fueron los que presentaron los mayores puntajes de riesgo TIMI, que sería lo esperado al ser una escala que predice la probabilidad de muerte a los 30 días, por lo que consideramos, al igual que Gines, que la mortalidad depende de la función ventricular, número de vasos afectados, inestabilidad eléctrica, grado de inflamación vascular, estado protrombótico, grado de comorbilidad, estado neurológico, así como de la experiencia del hospital que atiende al paciente en este tipo de casos. Por tal motivo es de vital importancia la capacitación médica continua, especialmente en este tipo de patologías con alta mortalidad y con una incidencia creciente.

\section{CONCLUSIONES}

La prevalencia de sospecha y diagnóstico clínico del síndrome coronario agudo en la Unidad de Medicina Familiar número 78, Guadalajara, Jalisco, es de $89.5 \%$, todos mostraron cuadro clínico de dolor torácico así como cambios electrocardiográficos con predominio del género femenino con una 
media de edad de 65.02 años y una desviación estándar de 12.033.

Con respecto a los factores de riesgo se detectó predominio de hipertensión, diabetes y sedentarismo; con base en la mortalidad tuvieron una incidencia muy baja de 3.5\% en comparación con la media nacional e internacional reportada por la OCDE. Consideramos que la mortalidad está relacionada con muchas causas, entre ellas, la experiencia del centro de atención médica que atiende al paciente.

El presente estudio servirá para investigaciones posteriores, ya que en urgencias de primer nivel de atención médica se han realizado pocos estudios, así como para implementar la educación continua de los médicos con el fin de mejorar la atención, diagnóstico y tratamiento de los pacientes, además ayudará a reducir la mortalidad de una de las patologías con mayor prevalencia y mortalidad.

Debido a los hallazgos en nuestro estudio consideramos importante apostar a la prevención tratando de disminuir el riesgo cardiovascular al reducir los factores de riesgo modificables y controlar de forma adecuada las patologías existentes, además de mejorar los procesos de identificación clínica, diagnóstico electrocardiográfico, tratamiento en primer nivel de atención y envío oportuno a segundo o tercer nivel.

\section{AGRADECIMIENTOS}

\author{
Al Instituto Mexicano del Seguro Social.
}

\section{Biblografía}

1. Alvarado RMA, Angulo CJM, Duarte SP, Fonseca GC, Monge BC, Quesada AC et al. Guía para diagnóstico y tratamiento de las personas con síndrome coronario agudo. San José, Costa Rica: EDNASSS-CCSS; 2013. pp. 1-72.

2. World Health Organization. WHO Mortality Database. Geneva, Switzerland: World Health Organization; 2015 [actualización 2017; acceso febrero 2019]. Disponible en: http://www.who.int/healthinfo/mortality_data/en/

3. Organización Mundial de la Salud. Prevention of cardiovascular disease. Guidelines for assessment and management of total cardiovascular risk. Ginebra: OMS; 2007.
4. Thygesen K, Alpert JS, Jaffe AS, Simoons ML, Chaitman BR, White HD et al. Third universal definition of myocardial infarction. Eur Heart J. 2012; 33 (20): 2551-2567.

5. Britto PF. Actualización en definición y manejo del infarto agudo del miocardio. Revista Peruana de Cardiología. 2008; 34 (1): 54-56.

6. Coll MY, Valladares CFJ, González RC. Infarto agudo de miocardio. Actualización de la Guía de Práctica Clínica. Rev Finlay. 2016; 6 (2): 170-190.

7. Moreno RNL. Modificación de los criterios de Sgarbossa para el diagnóstico de infarto agudo de miocardio en presencia de bloqueo de rama izquierda. Rev Fac Med. 2015; 63 (1): 151-154.

8. O'Donnel CJ, Elosua R. Factores de riesgo cardiovascular. Perspectivas derivadas del Framingham Heart Study. Rev Esp Cardiol. 2008; 61 (3): 299-310.

9. Sánchez CM, Moreno GGA, Marín GME, García OLH. Factores de riesgo cardiovascular en poblaciones jóvenes. Rev Salud Pública. 2009; 11 (1): 110-122.

10. Castellanos RR, Ferrer HI, Segura PLA, Ojeda MMR, Fernández RD. Infarto agudo del miocardio en pacientes jóvenes. Rev Arch Med Camagüey. 2014; 18 (6): 667-679.

11. De Abreu M, Cosarinsky L, Silberstein A, Mariana JA, Doval HC, Gagliardi JA et al. Características clínicas, angiográficas, estrategias terapéuticas y pronóstico de pacientes jóvenes con síndrome coronario agudo. Rev Argent Cardiol. 2013; 81: 22-30.

12. García CA, Sánchez DCJ, Martínez SC, Llamas EG, Cardona E, Barragán R et al. Guías clínicas para el manejo del infarto agudo del miocardio con elevación del segmento ST. Arch Cardiol Mex. 2006; 76 (3): 12-120.

13. Gines AS. Estratificación del riesgo en los síndromes coronarios agudos: un problema no resuelto. Rev Esp Cardiol. 2007; 60 (3): 23-30.

14. Instituto Nacional de Estadística y Geografía (INEGI). Estadísticas de mortalidad 2015. México: INEGI; 2015. [Acceso febrero 2019]. Disponible en: http://www.inegi.org. mx/est/contenidos/proyectos/registros/vitales/mortalidad/ tabulados/ConsultaMortalidad.asp

15. Organization for Economic Co-operation and Development (OECD). OECD Health Statistics 2013. Paris: OECD; 2013. [Acceso febrero 2019]. Disponible en: http://dx.doi. org/10.1787/health-data-en

16. Borrayo SG, Pérez RG, Martínez MOG, Almeida GE, Ramírez AE et al. Protocolo para atención de infarto agudo de miocardio en urgencias: Código infarto. Rev Med Inst Mex Seg Soc. 2017; 55 (2): 233-246.

17. Gasperin CO, Arteaga VA, Fuentes FI. Estimación de riesgo cardiovascular global en trabajadores de la delegación Veracruz, Sur [Tesis]. Córdoba, Veracruz: 2014. 